\title{
A Modificatory Screening Protocol for ARDS in Patients with Respiratory Support Based on SpO2 and $\mathrm{FiO2}$
}

\section{Yan Xia}

The First Affiliated Hospital of Wannan Medical College (Yijishan Hospital of Wannan Medical College)

\section{Qiancheng Xu}

The First Affiliated Hospital of Wannan Medical College (Yijishan Hospital of Wannan Medical College)

\section{Zhiyuan Guo}

The First Affiliated Hospital of Wannan Medical College (Yijishan Hospital of Wannan Medical College)

\section{Huijuan Zhang}

The First Affiliated Hospital of Wannan Medical College (Yijishan Hospital of Wannan Medical College)

\section{Yingya Cao}

The First Affiliated Hospital of Wannan Medical College (Yijishan Hospital of Wannan Medical College)

\section{Yupeng Qi}

The First Affiliated Hospital of Wannan Medical College (Yijishan Hospital of Wannan Medical College)

\section{Qun Chen}

The First Affiliated Hospital of Wannan Medical College (Yijishan Hospital of Wannan Medical College)

\section{Weihua Lu ( $\nabla$ lwh683@126.com )}

The First Affiliated Hospital of Wannan Medical College (Yijishan Hospital of Wannan Medical College)

\section{Research Article}

Keywords: ARDS, PaO2/fraction of inspired oxygen ratio, Sp02/fraction of inspired oxygen ratio, FiO2, Diagnose

Posted Date: August 4th, 2021

DOI: https://doi.org/10.21203/rs.3.rs-744649/v1

License: (c) (i) This work is licensed under a Creative Commons Attribution 4.0 International License. Read Full License 


\section{A Modificatory Screening Protocol for ARDS in Patients with Respiratory Support Based on $\mathrm{SpO}_{2}$ and $\mathrm{FiO}_{2}$}

Yan Xia, MD ${ }^{a}$; Qiancheng Xu, MD ${ }^{a, b}$; Zhiyuan Guo, MD ${ }^{a, b}$; Huijuan Zhang, $M D^{a, b}$; Yingya Cao, $M D^{a, b}$; Yupeng Qi, MD ${ }^{a, b}$; Qun Chen, MD ${ }^{a, b}$;Weihua Lu, MD ${ }^{a, b^{*}}$

a. Department of Critical Care Medicine, The First Affiliated Hospital of Wannan Medical College (Yijishan Hospital of Wannan Medical College), No.2, West road of Zheshan, Jinghu District, Wuhu, Anhui, 241000, China.

b. Anhui Province Clinical Research Center for Critical Care Medicine (Respiratory Disease)

* Corresponding Author. Weihua Lu: Department of Critical Care Medicine, The First Affiliated Hospital of Wannan Medical College (Yijishan Hospital of Wannan Medical College), No.2, West road of Zheshan, Jinghu District, Wuhu, Anhui, 241000, China. Tel:+86 0553-5739731. E-mail address: 1wh683@126.com

Email addresses for each co-author :

Yan Xia $\quad$ E-mail: 1275957591@qq.com

Qiancheng XuＥ-mail: xu871011@126.com

Zhiyuan Guo_E-mail:837765536@qq.com

Huijuan Zhang E-mail: 2689225788@qq.com

Yingya Cao_E-mail: caoyingya1990@126.com

Yupeng Qi $\quad$ E-mail: docqyp@163.com

Qun Chen E-mail: chenqun9532@163.com

Weihua Lu_E-mail: 1wh683@126.com

This manuscript text count for 4800 words. 


\begin{abstract}
Background: A large number of recent studies have confirmed that the pulse oximetric saturation $(\mathrm{SpO} 2)$ /fraction of inspired oxygen $(\mathrm{FiO} 2)$ ratio (SFR) correlate well with $\mathrm{PaO} 2 / \mathrm{FiO} 2$ ratio (PFR). However, whether SFR can replace PFR for the diagnosis and evaluate the severity of ARDS patients with advanced respiratory support is unclearly. The purpose of this study is to explore potential value of SFR ratio as a new diagnostic tool for ARDS by establishing new processes in patients who require high levels of oxygen support.
\end{abstract}

Methods: 341 patients were included in this study, SFR and PFR values were recorded in the same time. 161 patients were used to establish the model, and 180 patients were used to verify the validity of the model. 161 groups of data were divide into hypoxic group (group $\mathrm{H}$ ) and non-hypoxic group (group $\mathrm{N}$ ) according to whether $\mathrm{SpO}_{2}$ was greater than $97 \%$. For group $\mathrm{H}$, the regression equation was established to describe the relationship between SFR and PFR. and calculated the value of SFR when PFR is 300 . For group N, the correlation between each observation data and PFR were analyzed. Receiver operating characteristic (ROC) curve analysis was used to determine the diagnostic value of the index. Finally, a new diagnostic process was established for ARDS based on these results, and the reliability was verified with Berlin definition set as the gold standard for diagnosis and classification.

Results: For group H, the diagnostic linear equation is PFR $=0.9162 *$ SFR-21.39 $\left(\mathrm{R}^{2}=0.66, \mathrm{P}<0.0001\right.$ ). After calculation, when PFR is 300 , SFR is 352. For group N, There is a significant negative correlation between $\mathrm{FiO}_{2}$ and $\mathrm{PFR}((\mathrm{R}=-0.521, \mathrm{P}<0.0001))$. The AUC of using $\mathrm{FiO}_{2}$ to diagnose ARDS was $0.694(95 \% \mathrm{CI} 0.571 \sim 0.817, \mathrm{P}<$ 0.005 ). When the cutoff value of $\mathrm{FiO}_{2}$ was $39 \%$, the sensitivity was 0.838 and the specificity was 0.545 . Therefore, in this new diagnosis progress, when $\mathrm{SpO}_{2} \leq 97 \%$, if $\mathrm{SFR} \leq 352$, ARDS may exist; when $\mathrm{SpO}_{2}>97 \%$, if $\mathrm{FiO}_{2} \mathrm{~min}>39 \%$, there may be ARDS. The sensitivity, specificity, NPV, PPV, and accuracy of the new diagnosis progress for ARDS were $91.1 \%, 76.7 \%, 89.6 \%, 79.6 \%$, and $83.9 \%$, respectively. 
Conclusion: There are potential value of $\mathrm{SpO} 2$ and $\mathrm{FiO} 2$ as a noninvasive diagnostic tool for ARDS by new processes in patients who are already receiving high levels of oxygen support.

Keywords: ARDS; $\mathrm{PaO}_{2} /$ fraction of inspired oxygen ratio; $\mathrm{SpO}_{2} /$ fraction of inspired oxygen ratio; $\mathrm{FiO}_{2} ;$ Diagnose

\section{KEY POINTS}

Question: Is there any potential value of $\mathrm{SpO} 2$ and $\mathrm{FiO} 2$ as a diagnostic tool for ARDS Early Noninvasive Screening Protocol?

Findings: In this new diagnosis progress, when $\mathrm{SpO}_{2} \leq 97 \%$, if $\mathrm{SFR} \leq 352$, ARDS may exist; when $\mathrm{SpO}_{2}>97 \%$, if $\mathrm{FiO}_{2} \min >39 \%$, there may be ARDS. The sensitivity, specificity, NPV, PPV, and accuracy of the new diagnosis progress for ARDS were $91.1 \%, 76.7 \%, 89.6 \%, 79.6 \%$, and $83.9 \%$.

Meaning: There are potential value of $\mathrm{SpO}_{2}$ and $\mathrm{FiO}_{2}$ as a noninvasive diagnostic tool for ARDS.Continuous monitor $\mathrm{SpO}_{2}$ and $\mathrm{FiO}_{2}$ could enable clinicians to recognize ARDS timely and accurately. 


\section{Background}

Acute respiratory distress syndrome (ARDS) is devastating clinical syndromes with associated mortality more than $40 \%{ }^{[1]}$, which can be caused by a variety of etiologies. Although ARDS is a common fatal disease, In the LUNG SAFE study, the largest international cohort of patients with ARDS, however, there were two-thirds of patients who were delayed or missed the diagnosis of $\operatorname{ARDS}^{[2]}$. In the absence of accurate identification of ARDS, effective treatments have not been fully applied, including protective mechanical ventilation, fluid restriction, and prone positioning. PFR is the center of understanding, describing, and treating ARDS. PFR is not only used in the definition of ARDS, but also used to grade the severity of ARDS ${ }^{[3]}$.

In the absence of significant progress in the recognition and treatment of ARDS, an arterial blood gas analysis is required to determine the PFR of a patient, but patients who are not suspected of having ARDS may not undergo ABG examination, and frequent blood gas monitoring are also questioned. In addition, Bellani et $\mathrm{al}^{[4]}$. confirmed that even though the PFR is reached the standard, clinicians still could not recognize ARDS timely, due to $\mathrm{ABG}$ testing is too rare and discontinuous in one day, and parts of ARDS patients with contraindications of arterial puncture or in institutions without blood gas analysis conditions. Therefore, the development of approaches to enable more timely recognition has the potential to save lives.

In 2016, Riviello and his colleagues ${ }^{[5]}$ proposed that an SFR less than or equal to 315 can be used as the oxygenation standard under resource constraints, which is known as Kigali modification, and has been widely used. Brown et al. ${ }^{[6,7]}$ used the method of non-linear calculation to obtain accurate PFR in ARDS patients based on the SFR. However, the inclusion criteria for $\mathrm{SpO} 2$ in this study was less than $96 \%$. But, the patients who use ventilator or oxygen therapy to maintain $\mathrm{SpO} 2 \leq 96 \%$ may face with an early lung injury. If ignored, it may cause the disease to worsen ${ }^{[4]}$. At the same time, It is difficult to identify ARDS early when $\mathrm{SpO} 2>96 \%$ in patients with 
non-pulmonary causes of oxygen therapy. In particular, high flow oxygen therapy (HFNO) is currently widely used as an effective therapeutic support for acute respiratory failure in patients with ARDS, such as COVID-19 ${ }^{[8,9]}$. Studies have shown that the clinicians should focus on the severity of patients with lung injury to require high levels of oxygen support, suggesting the concept that the diagnosis of ARDS should not depend on the presence of an endotracheal tube or positive-pressure ventilation in adults ${ }^{[10]}$. Recently, Matthay et $\mathrm{al}^{[11]}$ recommend that the Berlin definition of ARDS be broadened to include patients treated with HFNO of at least 30 $\mathrm{L} / \mathrm{min}$ who fulfil the other criteria for the Berlin definition of ARDS and propose that this would be an advance for patients with lung injury who require high levels of oxygen support. In this case, from an epidemiological perspective, the inclusion of these patients would more comprehensively identify those with ARDS at an earlier stage of the syndrome, and would include more patients in resource-limited settings, as was recommended with the Kigali modification of the Berlin definition of ARDS. Therefore, the purpose of this study is to explore potential value of SFR ratio as a new diagnostic tool for ARDS again by establishing new processes in patients who require high levels of oxygen support.

\section{Methods}

\section{Study Design and Study Population}

This study is a prospective observational studies, and was conducted in Yijishan Hospital Affiliated to Wannan Medical College. The study was approved by the First Affiliated Hospital of Wannan Medical College and participating institution ethics committees (approval number: 2019-97).This is an observational study, arterial blood samples and vital signs monitoring are parts of its standard treatment.The requirement for written informed consent was waived by the IRB. This clinical trial was registered in Chinese Clinical Trial Registry (ChiCTR,ChiCTR2000029217) by Weihua Lu in 19 January 2020, which was prior to patient enrollment. 
The inclusion critera is that patients who are admitted to the Department of Critical Care Medicine in our hospital from August to October 2020 and require arterial blood gas analysis and ECG monitoring due to their condition. Patients with contraindications to arterial puncture, methemoglobinemia and other reasons that affect data collection are excluded. All the arterial blood samples were analyzed using the blood gas analyzer (Danish Raydu ABL800 FLEX)within one minute after the blood collection, and the monitor (China Mindray iPM-12) was used to record the $\mathrm{SpO}_{2}$ and vital signs data immediately after the blood collection. To ensure the accuracy of the data, the blood oxygen saturation sensor is placed on a clean thumb; the blood oxygen saturation waveform is stable; there is no posture change or sputum suction operation at least 10 minutes before the measurement.

\section{Data Collection}

Demographic and clinical data of the included patients were collected: gender, age, etc. Immediate percutaneous peripheral blood oxygen saturation $\left(\mathrm{SpO}_{2}\right)$, inspired oxygen concentration $\left(\mathrm{FiO}_{2}\right)$, arterial oxygen partial pressure $\left(\mathrm{PaO}_{2}\right)$, respiratory rate (RR) were recorded, $\mathrm{SpO}_{2} / \mathrm{FiO}_{2}$ ratio (SFR) and $\mathrm{PaO}_{2} / \mathrm{FiO}_{2}$ ratio (PFR) values were calculated according to the above information.

\section{Statistical Analysis}

All normal distribution measurement data are represented by $(\overline{\mathrm{X}} \pm \mathrm{s})$, non-normal distribution count data are represented by median (P25, P75), and count data are represented by example(\%).The two independent sample t test was used for the comparison between the normal distribution measurement data, the Mann-Whitney U test was used for the comparison between the nonnormal distribution measurement data, and the $\chi^{2}$ test was used for the comparison between the count data. The test level is two-sided $\alpha=0.05, \mathrm{P}<0.05$, which indicates that the difference is statistically significant. 
Establishment of the New Diagnosis Tool: 341 patients were included in this study, SFR and PFR values recorded in the same time. 161 patients were used to establish the model, and 180 patients were used to verify the validity of the model. 161 groups of data were divide into hypoxic group (group H) and non-hypoxic group (group N) according to whether $\mathrm{SpO}_{2}$ was greater than $97 \%$. For group $\mathrm{H}$, the regression equation was established to describe the relationship between SFR and PFR. and calculated the value of SFR when PFR is 300 . For group N, the correlation between each observation data and PFR were analyzed and choose the value index. Receiver operating characteristic (ROC) curve analysis was used to determine the diagnostic value of the index. Finally, use SFR and the index to establish a new diagnostic process for ARDS. After determining the new diagnostic process, the Berlin definition is used as the gold standard to calculate the NPV, PPV, accuracy, sensitivity and specificity of the new diagnostic method.

Verify the Reliability of the New Diagnosis Tool: At the same time, another group of 180 ICU patients requiring blood gas analysis were collected to verify the reliability of the diagnosis tool. The Berlin definition is used as the gold standard to diagnose the presence or absence of ARDS. According to the Berlin definition grading standard, the corresponding SFR values were calculated with PFR of 100, 200 and 300 as the cutoff values, and the accuracy of the new method used for grading was verified.

\section{Results}

Of the 375 patients who needed arterial blood gas analysis in the Department of Intensive Care Medicine of our hospital , 34 were excluded due to their inaccurate data collection. Finally, there were 341 patients included in this study, including 160 males $(46.9 \%)$ and 181 females $(53.1 \%)$. The subjects were randomly divided into two cohort(Fig 1). The basic information of the research objects is shown in Table 1.

\section{Establishment of a New Diagnosis Process}




\section{Linear equations of group $\mathbf{H}$}

1.1 The correlation between SFR and PFR Finally, there are 87 cases in group H. because the data is not normally distributed, Spearman correlation is used to evaluate the correlation between SFR and PFR. SFR and SFR have a strong positive correlation $\left(\mathrm{R}^{2}=0.66, P<0.001\right)$.

\subsection{Establishment of Linear Regression}

To further clarify the correlation between SFR and PFR. The Linear regression between SFR and PFR was performed. linear regression equation indicated that there is a strong linear relationship between SFR and PFR $\left(\mathrm{R}^{2}=0.66, \mathrm{P}<0.0001\right)$. the linear regression equation as fellows:

$$
\mathrm{PFR}=0.9162 * \mathrm{SFR}-21.39
$$

The linear relationship diagram is shown in Fig 2.

2. The correlation between $\mathbf{F i O}_{2}$ and PFR in group $\mathbf{N}$ There are 74 cases in group N. All the $\mathrm{SpO}_{2}$ more than 97\%. correlation analysis shows than only the $\mathrm{FiO}_{2}$ and PFR have a significant negative correlation $(\mathrm{R}=-0.521, \mathrm{P}<0.0001)$. The receiver operation characteristic curve( $\mathrm{ROC}$ curve) was used to investigate the value of $\mathrm{FiO}_{2}$ in diagnosis of ARDS, The AUC of using $\mathrm{FiO}_{2}$ to diagnose ARDS was 0.694 (95\%CI $0.571 \sim 0.817, \mathrm{P}<0.005)$. the best cutoff value of $\mathrm{FiO}_{2}$ was $39 \%$, the sensitivity was 0.838 and the specificity was 0.545 . The ROC curve is shown in Fig 3 .

3. Establish a New Diagnosis Tool Based on the above results, we set up a new diagnosis process as follows (Fig 4): when a patients with suspicious ARDS, the $\mathrm{SpO}_{2} \leq 97 \%$, calculate SFR according to the formula: $\mathrm{SFR}=\mathrm{SpO}_{2} / \mathrm{FiO}_{2}$, if $\mathrm{SFR} \leq 352$, ARDS may exist. When $\mathrm{SpO}_{2}>97 \%$, if high concentration inhaled oxygen is required, ie $\mathrm{FiO}_{2 \min }>39 \%$, ARDS may exist. 


\section{Reliability of the New Diagnostic Method}

A total of 180 patients in the intensive care medicine department of our hospital who needed arterial blood gas analysis due to their condition were included in the verification group, There are both 90 cases with $\mathrm{SpO}_{2} \leq 97 \%$ and $\mathrm{SpO}_{2}>97 \%$. With the Berlin definition as the gold standard, the sensitivity, specificity, NPV, PPV, and accuracy of the new diagnostic tool were $91.1 \%, 76.7 \%, 89.6 \%, 79.6 \%$, and $83.9 \%$.

\section{The utility of different SFR cutoffs for ARDS prediction}

According to the established linear regression equation $(\mathrm{PFR}=0.9162 * \mathrm{SFR}$ -21.39), when PFR is 300, SFR is 352, when PFR is 200, SFR is 241, and when PFR is 100 , SFR is 132. ROC curves were used to investigate the value of different SFR cut-off value in diagnosis of ARDS, The 3 presented cutoffs demonstrated good specificity $(98.5 \%, 62.8 \%$, and $93.1 \%$, respectively) and sensitivity $(61.1 \%, 89.2 \%$, and $63.4 \%$, respectively). And the PPV and NPV were also high. The utility of the new method for ARDS classification was verified in Table 2.

\section{Discussion}

Our study reinforce the value of using $\mathrm{SpO}_{2}$ and $\mathrm{FiO}_{2}$ as an noninvasive diagnostic tool for ARDS, We also set up a new diagnosis process base on $\mathrm{SpO}_{2}$ and $\mathrm{FiO}_{2}$, when a patients suspicious with $\mathrm{ARDS}$, the $\mathrm{SpO}_{2} \leq 97 \%$, calculate SFR according to the formula: $\mathrm{SFR}=\mathrm{SpO}_{2} / \mathrm{FiO}_{2}$, if $\mathrm{SFR} \leq 352$, ARDS may exist; when $\mathrm{SpO}_{2}>97 \%$, if high concentration inhaled oxygen is required, ie $\mathrm{FiO}_{2} \min >39 \%$, ARDS may exist. Meanwhile, We verified the accuracy of the model, The model has a very high sensitivity and specificity. Our finding could to enable clinicians to recognize ARDS timely and accurately, especially in developing countries which lacking sufficient medical resources. However, saturation-based measurements are easily available in relatively resource-poor settings. 
The establishment of the Berlin definition in 2012 made the diagnosis and classification of ARDS more accurate and standardized ${ }^{[12]}$. However, repeated blood draws in the ICU environment may lead to iatrogenic anemia, the need for blood transfusions, an increased risk of infection, and an increase in hospital costs ${ }^{[13]}$. In addition, as mentioned earlier, many underdeveloped regions and countries may lack the conditions for arterial blood gas. Therefore, a large number of recent studies ${ }^{[6,14,15]}$ have confirmed that SFR can be used to evaluate ARDS patients. For example, Kigali University Teaching Hospital proposed that if arterial blood gas and chest $\mathrm{X}$-ray are difficult to achieve, $\mathrm{SpO}_{2} / \mathrm{FiO}_{2}$ ratio and lung ultrasound $\mathrm{Can}$ be used as a substitute for diagnosis of $\operatorname{ARDS}^{[16]}$.

This study confirmed that there is a strong correlation between SFR and PFR, and it is statistically significant $(\mathrm{r}=0.873, \mathrm{P}<0.0001)$, which means that this non-invasive methods can be used to estimate $\mathrm{PaO}_{2}$ and oxygenation index in clinical work. This is similar to the results of Katherine DesPrez, BA ${ }^{[14]}$. They proposed that SFR can be used as a substitute for PFR and provide noninvasively prognostic information and assess the severity of ARDS. The linear regression equation of the relationship between SFR and PFR calculated in this study is PFR $=0.9162 *$ SFR-21.39, which has a higher correlation coefficient, which shows that the formula in this study may be more in line with the linear relationship between SFR and PFR. The oxygenation index calculated by this formula may be more accurate. When $\mathrm{SpO}_{2}>97 \%$, statistical analysis shows that $\mathrm{FiO}_{2}$ is significantly correlated with $\mathrm{PFR}((\mathrm{R}=-0.521, \mathrm{P}<0.0001))$. In clinical applications, if the blood oxygen saturation is within the normal range due to the excessively high inspired oxygen concentration ,there maybe ARDS too ${ }^{[17]}$.

In the oxygen dissociation curve ${ }^{[18]}$, it can be seen that the curve drawn by the arterial blood oxygen saturation with the change of the arterial blood oxygen partial pressure is divided into three sections: upper, middle and lower. In the middle and lower part of the curve, the curve is steep, and $\mathrm{SaO}_{2}$ changes greatly with $\mathrm{PaO}_{2}$, which is conducive to hemoglobin releasing oxygen for tissue utilization. Therefore, separating 
the data with $\mathrm{SpO}_{2} \leq 96 \%$ for linear regression, the correlation between the two is higher than before. In the upper part, the curve is flat, and $\mathrm{SaO}_{2}$ changes little with $\mathrm{PaO}_{2}$. Therefore, when $\mathrm{SpO}_{2}>97 \%$, the PFR calculated by the linear formula is not accurate. Through statistical analysis, there is a significant correlation between $\mathrm{FiO}_{2}$ and PFR at this time. when $\mathrm{FiO}_{2}$ more than $39 \%$. The sensitivity of using $\mathrm{FiO}_{2}$ to diagnose ARDS is high, which is conducive to the early warning of ARDS. In the early research of Rice TW et al. ${ }^{[19]}$, they also explored the relationship between $\mathrm{SpO}_{2}$ and $\mathrm{PaO}_{2}$ and concluded that it is similar to this experiment, but they only analyzed the data of $\mathrm{SpO}_{2} \leq 97 \%$. They pointed out in the discussion that in the oxygen dissociation curve, the slope of $\mathrm{SpO}_{2}$ and $\mathrm{PaO}_{2}$ in the saturated state is almost zero, and a large change in $\mathrm{PaO}_{2}$ may cause little or no change in $\mathrm{SpO}_{2}$. So it excludes patients' data with high oxygen saturation. However, in clinical work, patients who rely on high concentration oxygen support,even though the monitor indicates that the blood oxygen saturation is normal, they may already have ARDS ${ }^{[20]}$. In this case, if they are not detected and treated early, which may cause illness deterioration. In our research, if the minimum $\mathrm{FiO}_{2}\left(\mathrm{SpO}_{2}>97 \%\right)$ is greater than $39 \%$, the patient may have early ARDS. The results of this study are of great significance to avoid ignoring the occurrence and development of ARDS. On the basis of the research results, a flow chart was drawn for the rapid diagnosis tool of high-risk patients (Fig3). Taking Berlin's definition as the gold standard for diagnosis, the diagnosis of the new diagnostic process has high sensitivity and specificity, indicating that the new diagnostic tool is suitable for early recognition of ARDS. However, a large sample of multiple centers is still needed for further verification.

However, it is worth mentioning that not all patients are suitable for condition assessment using this method. For example, in the case of carbon monoxide poisoning, peripheral circulatory failure, etc., it may not be possible to use finger pulse oxygen to monitor blood oxygen saturation, and blood gas analysis is the only way to rule out this disease ${ }^{[21]}$.Transcutaneous blood oxygen saturation technology transmits two wavelengths of light (usually $660 \mathrm{~mm}$ and $940 \mathrm{~mm}$ ) through the tissue to measure the 
absorbance change of each wavelength over time. The absorption of light by the tissue is cyclic, which is due to the circulation of the heart and the resulting pulsation of arterial blood into the tissue bed. However, $\mathrm{COHb}$ and $\mathrm{O}_{2} \mathrm{Hb}$ have similar absorption characteristics at $660 \mathrm{~mm}$. Therefore, the percutaneous oxygen saturation $\left(\mathrm{SpO}_{2}\right)$ of patients with carb monoxide and peripheral circulatory failure must not be an accurate measurement of $\mathrm{O}_{2} \mathrm{Hb}^{[22]}$. A recent observational study also confirmed that in patients using VV-ECMO, transcutaneous oxygen saturation is a poor oxygenation test. Due to the increase in $\mathrm{COHb}$ levels, as the VV-ECMO support time increases, Its reliability is reduced ${ }^{[23]}$.This situation can also be seen in long-term smokers. Early studies have confirmed that the $\mathrm{COHb}$ content in the blood of long-term smokers will increase by $15 \%{ }^{[24]}$. Recent study has shown that carboxyhemoglobin saturation (SpCO) baseline levels are significantly higher in long-term smokers than in nonsmokers ${ }^{[25]}$.Under these above circumstances, only blood gas analysis and percutaneous carbon monoxide analyzer can judge the patient's true oxygenation situation comprehensively.

Moreover, the new procedures should not obscure the need to exclude left heart failure or primary lung disease (such as autoimmune disease or acute eosinophilic pneumonia). The expanded definition will enable the diagnosis of ARDS to be more widely applicable and will help identify patients in the early stages of the syndrome as well as patients with severe clinical lung injury who are already receiving high levels of oxygen support. The implementation may improve patient clinical practice and clinical outcomes.

There are some shortcomings in this study. First of all, it is a single-center observational study with a small sample size, so the new diagnostic tool needs to be verified by a multi-center large-sample study. In addition, we explored the linear relationship between SFR and PFR without considering the influence of other factors on the oxygen dissociation curve, such as $\mathrm{pH}, \mathrm{CO}_{2}$, etc.. This is because the purpose of this study is to explore the general relationship between the two, so that diagnostic 
tools can be used even when these confounders are unknown. Similarly, we did not conduct separate studies on different ventilation modes, such as ventilation mode and PEEF,etc.. Finally, this study only verified the efficacy of this new diagnostic tool, and did not explore its correlation with the prognosis of ARDS patients, which could be explored in later studies.

\section{Conclusions}

Measurement of the $\mathrm{SpO}_{2}$ and $\mathrm{FiO}_{2}$ performed as well as $\mathrm{PaO}_{2}$ in diagnosis of ARDS and grade the severity, while avoiding invasive arterial blood gas monitoring. Meanwhile, Continuous monitor $\mathrm{SpO}_{2}$ and $\mathrm{FiO}_{2}$ could enable clinicians to recognize ARDS timely and accurately, especially for patients with lung injury who require high levels of oxygen support. 


\begin{abstract}
Abbreviations
$\mathrm{ARDS}=$ acute respiratory distress syndrome; $\mathrm{SFR}=\mathrm{SpO} 2 /$ fraction of inspired oxygen ratio; $\mathrm{PFR}=\mathrm{PaO} 2 /$ fraction of inspired oxygen ratio; $\mathrm{ROC}=\mathrm{Receiver}$ operating characteristic; $\mathrm{AUC}=\mathrm{Area}$ Under Curve; $\mathrm{ABG}=\mathrm{Arterial}$ blood gas; $\mathrm{ICU}=$ Intensive Care Unite.
\end{abstract}

\title{
Acknowledgements
}

Thanks to each of the study participants for contributing to this article.

\section{Disclosures}

Name: Weihua Lu, MD

Contribution: This author was responsible for the conceptualization of the study and the revision and approval of this manuscript.

Name: Qiancheng Xu, MD and Yan Xia, MD

Contribution: These authors were participated in the design and drafted the manuscript.

Name: Zhiyuan Guo, MD and Huijuan Zhang, MD

Contribution: These authors collected data and were responsible for its accuracy.

Name: Yingya Cao, Yupeng Qi, MD and Qun Chen, MD

Contribution:These authors helped to revise the manuscript.

All authors contributed to the data analysis and interpretation. All authors read and approved the final manuscript.

\section{Funding}

This work was supported, in part, by the Anhui Provincial Special Project of Central Government Guiding Local Science and Technology Development of China (201907d07050001). and Funding of "Peak" Training Program for Scientific Research of Yijishan Hospital, Wannan Medical College(GF2019J03, GF2019G08). 


\section{Availability of data and materials}

The data used to support the findings of this study are available from the corresponding author upon reasonable request.

\section{Ethics approval and consent to participate}

The study was approved by the First Affiliated Hospital of Wannan Medical College and participating institution ethics committees (approval number: 2019-97).

\section{Trail registration}

ChiCTR. ChiCTR2000029217. Registered in 19 January 2020, http://www.chictr.org.cn/showproj.aspx?proj=43364

\section{Consent for publication}

All authors have approved the manuscript and its publication.

\section{Competing interests}

The authors have no competing interests. 


\section{References}

[1] Ike JD, Kempker JA, Kramer MR, Martin GS. The Association Between Acute Respiratory Distress Syndrome Hospital Case Volume and Mortality in a U.S. Cohort, 2002-2011. Crit Care Med. 2018. 46(5): 764-773.

[2] Bellani G, Laffey JG, Pham T, et al. Epidemiology, Patterns of Care, and Mortality for Patients With Acute Respiratory Distress Syndrome in Intensive Care Units in 50 Countries. JAMA. 2016. 315(8): 788-800.

[3] Ferguson ND, Fan E, Camporota L, et al. The Berlin definition of ARDS: an expanded rationale, justification, and supplementary material. Intensive Care Med. 2012. 38(10): 1573-82.

[4] Bellani G, Pham T, Laffey JG. Missed or delayed diagnosis of ARDS: a common and serious problem. Intensive Care Med. 2020. 46(6): 1180-1183.

[5] Riviello ED, Buregeya E, Twagirumugabe T. Diagnosing acute respiratory distress syndrome in resource limited settings: the Kigali modification of the Berlin definition. Curr Opin Crit Care. 2017. 23(1): 18-23.

[6] Brown SM, Grissom CK, Moss M, et al. Nonlinear Imputation of Pao2/Fio2 From Spo2/Fio2 Among Patients With Acute Respiratory Distress Syndrome. Chest. 2016. 150(2): 307-13.

[7] Brown SM, Duggal A, Hou PC, et al. Nonlinear Imputation of PaO2/FIO2 From SpO2/FIO2 Among Mechanically Ventilated Patients in the ICU: A Prospective, Observational Study. Crit Care Med. 2017. 45(8): 1317-1324.

[8] Calligaro GL, Lalla U, Audley G, et al. The utility of high-flow nasal oxygen for severe COVID-19 pneumonia in a resource-constrained setting: A multi-centre prospective observational study. EClinicalMedicine. 2020. 28: 100570.

[9] Perkins GD, Couper K, Connolly B, et al. RECOVERY- Respiratory Support: Respiratory Strategies for patients with suspected or proven COVID-19 respiratory failure; Continuous Positive Airway Pressure, High-flow Nasal Oxygen, and standard care: A structured summary of a study protocol for a randomised controlled trial. Trials. 2020. 21(1): 687.

[10] García-de-Acilu M, Marin-Corral J, Vázquez A, et al. Hypoxemic Patients With Bilateral Infiltrates Treated With High-Flow Nasal Cannula Present a Similar Pattern of Biomarkers of Inflammation and Injury to Acute Respiratory Distress Syndrome Patients. Crit Care Med. 2017. 45(11): 1845-1853.

[11] Matthay MA, Thompson BT, Ware LB. The Berlin definition of acute respiratory distress syndrome: should patients receiving high-flow nasal oxygen be included. Lancet Respir Med. 2021 .

[12] ARDS Definition Task Force, Ranieri VM, Rubenfeld GD, et al. Acute respiratory distress syndrome: the Berlin Definition. JAMA. 2012. 307(23): 2526-33.

[13] Gopalratnam K, Forde IC, O'Connor JV, Kaufman DA. Less Is More in the ICU: Resuscitation, Oxygenation and Routine Tests. Semin Respir Crit Care Med. 2016. 37(1): 23-33.

[14] DesPrez K, McNeil JB, Wang C, Bastarache JA, Shaver CM, Ware LB. Oxygenation Saturation Index Predicts Clinical Outcomes in ARDS. Chest. 2017. 152(6): 1151-1158.

[15] Batchinsky AI, Wendorff D, Jones J, et al. Noninvasive SpO2/FiO2 ratio as surrogate for $\mathrm{PaO} 2 / \mathrm{FiO} 2$ ratio during simulated prolonged field care and ground and high-altitude evacuation. J Trauma Acute Care Surg. 2020. 89(2S Suppl 2): S126-S131.

[16] Chen W, Janz DR, Shaver CM, Bernard GR, Bastarache JA, Ware LB. Clinical Characteristics and Outcomes Are Similar in ARDS Diagnosed by Oxygen Saturation/Fio2 Ratio Compared With Pao2/Fio2 Ratio. Chest. 2015. 148(6): 1477-1483.

[17] Martin DS, Grocott MP. Oxygen therapy in critical illness: precise control of arterial oxygenation and 
permissive hypoxemia. Crit Care Med. 2013. 41(2): 423-32.

[18] Collins JA, Rudenski A, Gibson J, Howard L, O'Driscoll R. Relating oxygen partial pressure, saturation and content: the haemoglobin-oxygen dissociation curve. Breathe (Sheff). 2015. 11(3): 194-201.

[19] Rice TW, Wheeler AP, Bernard GR, et al. Comparison of the $\mathrm{SpO} 2 / \mathrm{FIO} 2$ ratio and the $\mathrm{PaO} 2 / \mathrm{FIO} 2$ ratio in patients with acute lung injury or ARDS. Chest. 2007. 132(2): 410-7.

[20] Meyhoff CS, Staehr AK, Rasmussen LS. Rational use of oxygen in medical disease and anesthesia. Curr Opin Anaesthesiol. 2012. 25(3): 363-70.

[21] Hampson NB. Pulse oximetry in severe carbon monoxide poisoning. Chest. 1998. 114(4): 1036-41.

[22] Ralston AC, Webb RK, Runciman WB. Potential errors in pulse oximetry. I. Pulse oximeter evaluation. Anaesthesia. 1991. 46(3): 202-6.

[23] Nisar S, Gibson CD, Sokolovic M, Shah NS. Pulse Oximetry Is Unreliable in Patients on Veno-Venous Extracorporeal Membrane Oxygenation Caused by Unrecognized Carboxyhemoglobinemia. ASAIO J. 2020 .

[24] Castleden, C., M., Cole, P., V. Variations in Carboxyhaemoglobin Levels in Smokers. BMJ. 1974.

[25] Schimmel J, George N, Schwarz J, Yousif S, Suner S, Hack JB. Carboxyhemoglobin Levels Induced by Cigarette Smoking Outdoors in Smokers. J Med Toxicol. 2018. 14(1): 68-73. 
Table 1. Characteristics of subjects

\begin{tabular}{ll}
\hline \multicolumn{1}{c}{ Characteristics } & \multicolumn{1}{c}{$\mathbf{n}=\mathbf{3 4 1}$} \\
\hline Sex, n (\%) & $160(46.9)$ \\
Male & $181(53.1)$ \\
Female & $57 \pm 10.11$ \\
Mean body weight (range), kg & $48(19-78)$ \\
Age (range),years old & $45.7(20-100)$ \\
Mean FiO2 (range), \% & $93.84(35.4-230)$ \\
Mean PaO2 (range), mmHg & $94.82(63-100)$ \\
Mean SpO2 (range), \% & $226.57(48.9-540)$ \\
Mean PFR (range) & $226.40(73.8-456)$ \\
Mean SFR (range) &
\end{tabular}

Table 2. Utility of SFR for ARDS classification $\left(\mathrm{SpO}_{2} \leq \mathbf{9 7 \%}\right)$

\begin{tabular}{cccccc}
\hline SFR & Sensitivity(\%) & Specificity(\%) & PPV(\%) & NPV(\%) & + LR,-LR \\
\hline$<132$ & 61.1 & 98.5 & 84.6 & 95 & $40.73^{\mathrm{a}}, 0.39$ \\
$<241$ & 89.2 & 62.8 & 74.2 & 83 & $2.39,0.17$ \\
$<352$ & 63.4 & 93.1 & 82.5 & 83.3 & $9.18,0.04^{\mathrm{b}}$ \\
\hline
\end{tabular}

a:The likelihood of diagnosing ARDS is significantly increased when the positive likelihood ratio is greater than 10. ${ }^{\mathbf{b}}$ :The likelihood of excluding ARDS is significantly increased when the negative likelihood ratio is less than 0.1 . 


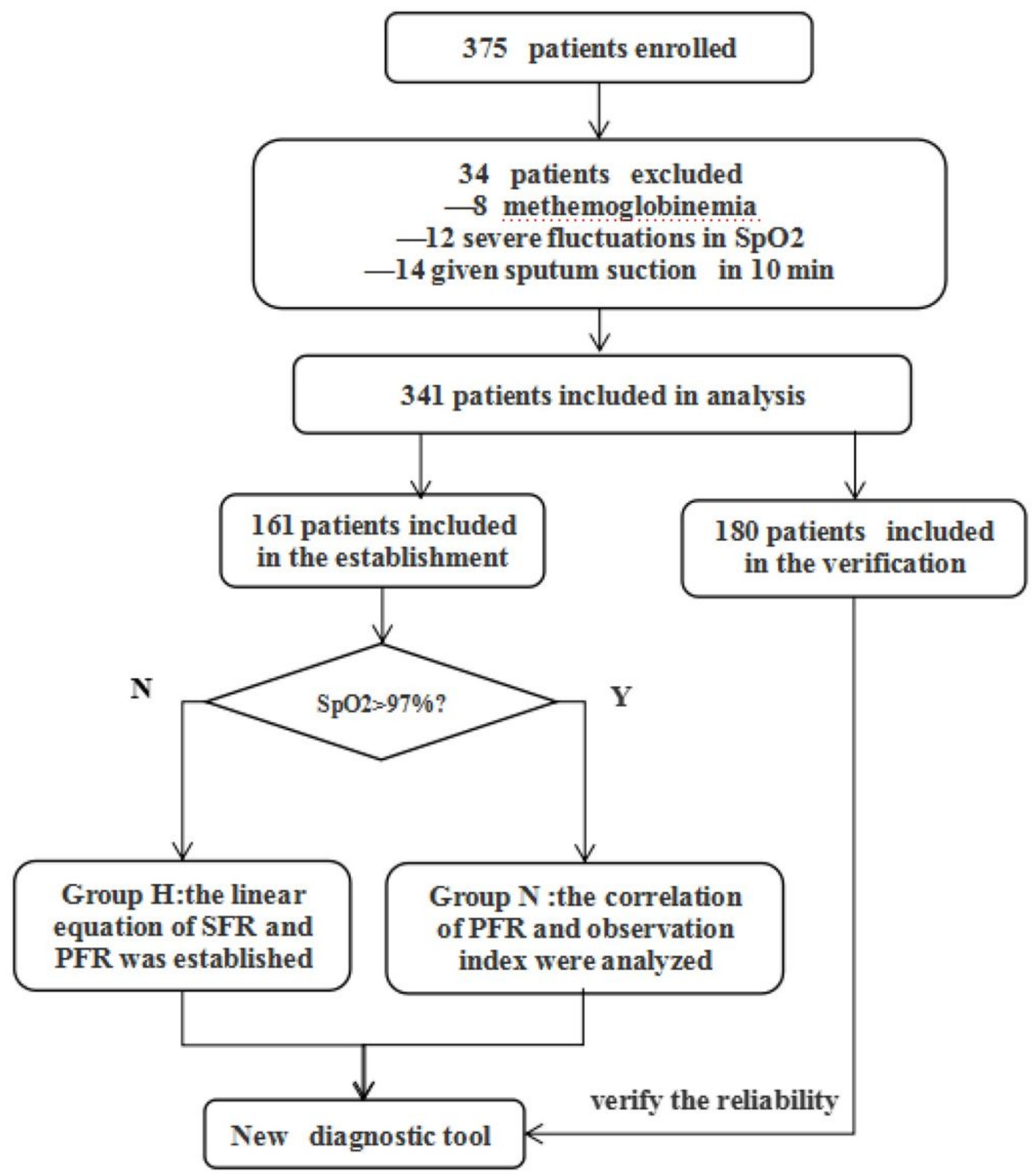

Figure 1

Finally, there were 341 patients included in this study, including 160 males (46.9\%) and 181 females (53.1\%). The subjects were randomly divided into two cohort(Fig 1) 


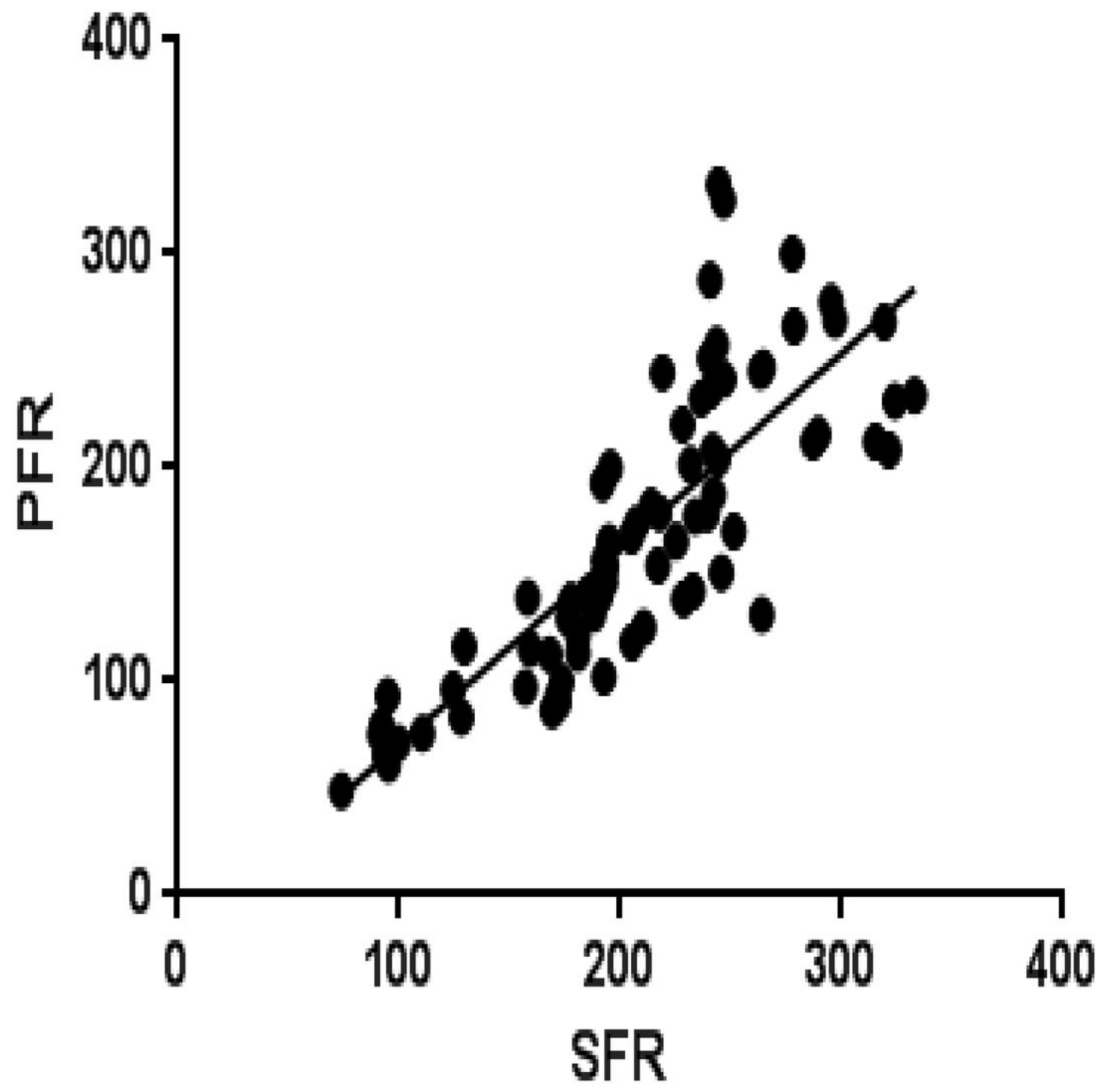

Figure 2

The linear relationship diagram is shown in Fig 2. 


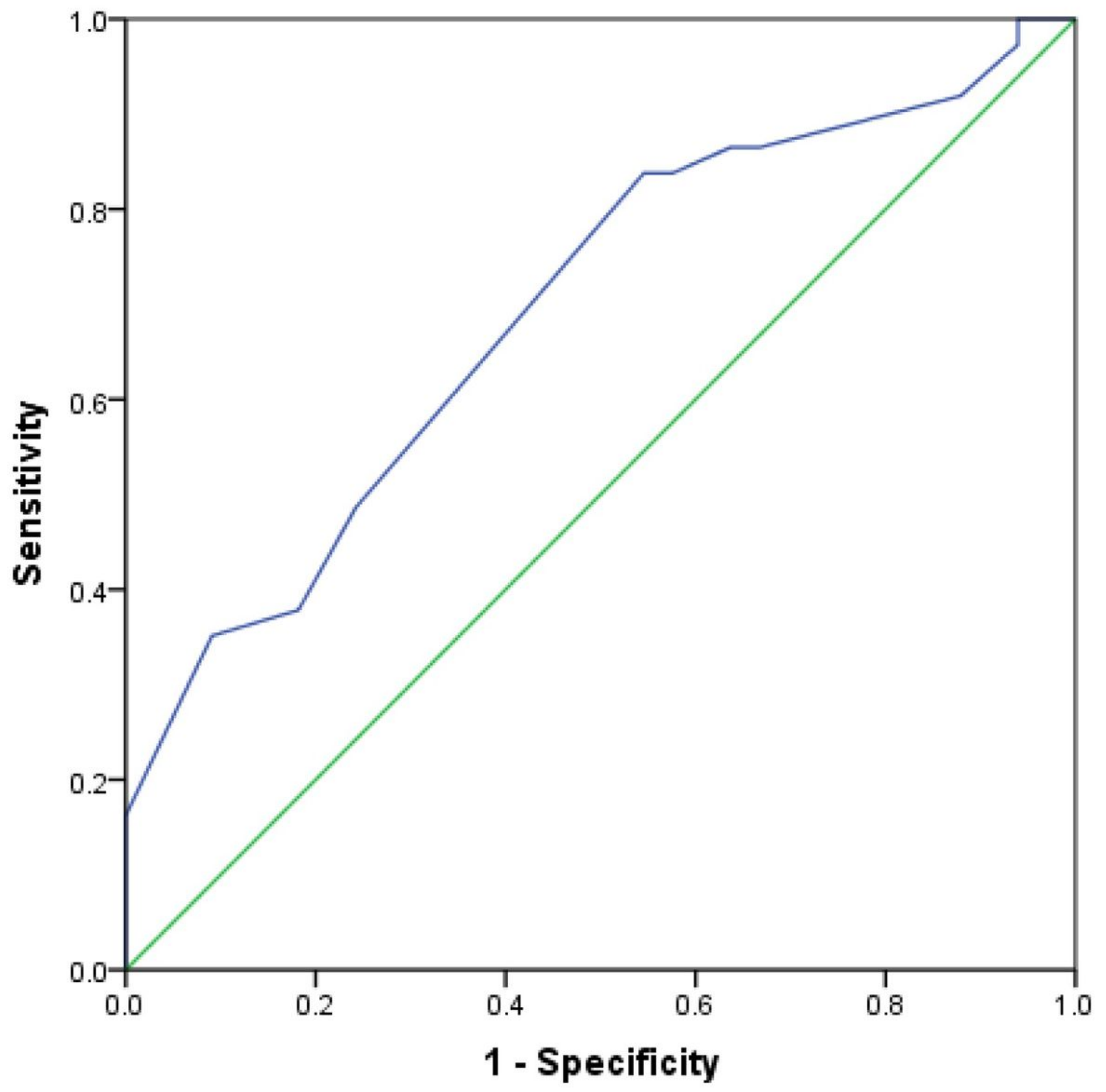

Figure 3

the best cutoff value of $\mathrm{FiO} 2$ was $39 \%$, the sensitivity was 0.838 and the specificity was 0.545 . The ROC curve is shown in Fig 3. 


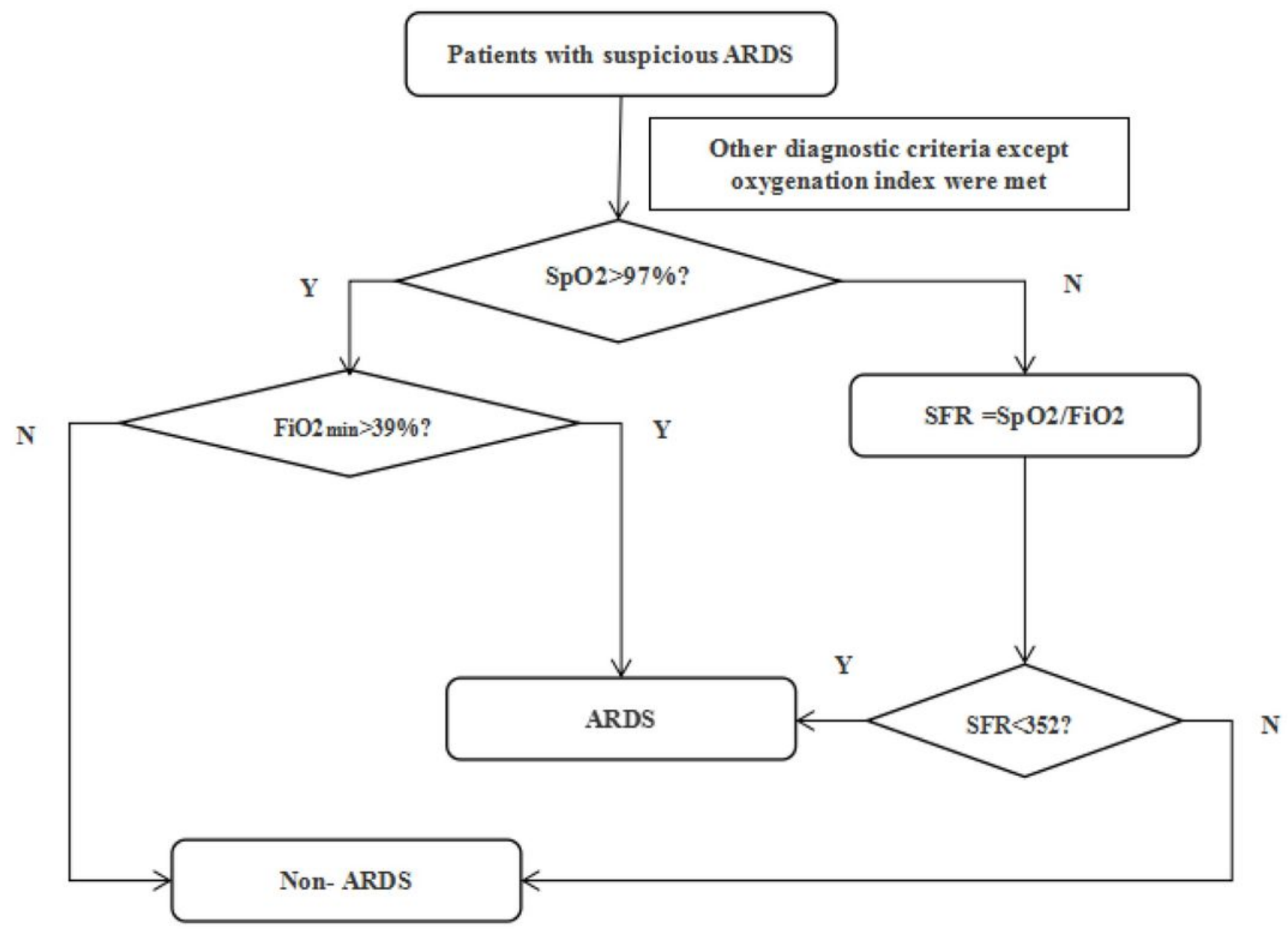

Figure 4

Based on the above results, we set up a new diagnosis process as follows (Fig 4)

\section{Supplementary Files}

This is a list of supplementary files associated with this preprint. Click to download.

- STARDchecklist.docx 